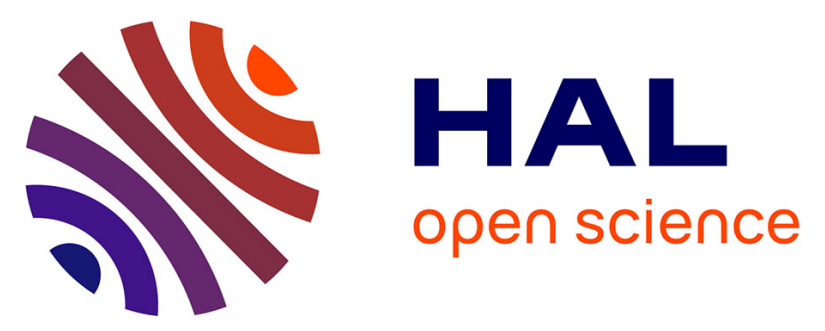

\title{
LA FLORE FOSSILE DU SYSTÈME TRAVERTINEUX DU SERRE DE MONTDENIER (ALPES DE HAUTE PROVENCE, FRANCE) : UN NOUVEAU JALON DANS L'HISTOIRE DE LA VÉGÉTATION HOLOCÈNE DES ALPES DU SUD
}

Paul Roiron, Adam A Ali, Jean-Louis Guendon, Marie-Eve Migueres, Serge Muller, Vincent Ollivier

\section{- To cite this version:}

Paul Roiron, Adam A Ali, Jean-Louis Guendon, Marie-Eve Migueres, Serge Muller, et al.. LA FLORE FOSSILE DU SYSTÈME TRAVERTINEUX DU SERRE DE MONTDENIER (ALPES DE HAUTE PROVENCE, FRANCE): UN NOUVEAU JALON DANS L'HISTOIRE DE LA VÉGÉTATION HOLOCÈNE DES ALPES DU SUD. Quaternaire, 2006, 10.4000/quaternaire.786 . hal-01766599

\author{
HAL Id: hal-01766599 \\ https://hal.science/hal-01766599
}

Submitted on 25 May 2018

HAL is a multi-disciplinary open access archive for the deposit and dissemination of scientific research documents, whether they are published or not. The documents may come from teaching and research institutions in France or abroad, or from public or private research centers.
L'archive ouverte pluridisciplinaire HAL, est destinée au dépôt et à la diffusion de documents scientifiques de niveau recherche, publiés ou non, émanant des établissements d'enseignement et de recherche français ou étrangers, des laboratoires publics ou privés. 


\title{
LA FLORE FOSSILE DU SYSTÈME TRAVERTINEUX DU SERRE DE MONTDENIER (ALPES DE HAUTE PROVENCE, FRANCE) : UN NOUVEAU JALON DANS L'HISTOIRE DE LA VÉGÉTATION HOLOCÈNE DES ALPES DU SUD
}

\author{
Paul ROIRON ${ }^{1}$, Adam A. ALI ${ }^{1}$, Jean-Louis GUENDON², Marie-Eve MIGUERES ${ }^{1}$, \\ Serge D. MULLER ${ }^{3} \&$ Vincent OLLIVIER ${ }^{2}$
}

\begin{abstract}
RÉSUMÉ
Le système travertineux du Serre de Montdenier (alt. 1200 m) présente des massifs contenant des macrorestes végétaux (empreintes foliaires et charbons) et une tourbière minérotrophe alcaline. L'analyse des séquences travertineuses datées par U/Th et ${ }^{14} \mathrm{C}$ a permis de reconstituer la structure et la composition de la flore ligneuse locale au début de l'Holocène. Le paysage du vallon de Mouresse se présentait alors sous la forme d'une mosaïque de chênaies caducifoliées mésophiles, de zones ouvertes à Amelanchier ovalis et Berberis vulgaris, et de ripisylves à Alnus glutinosa, Salix spp. et Vitis vinifera subsp. sylvestris. Plusieurs de ces espèces ont disparu de la végétation locale, actuellement caractérisée par des matorrals à Buxus sempervirens et des reboisements de Pinus nigra subsp. nigra. La tourbière minérotrophe, qui recouvre la partie sommitale du système travertineux, se développa il y a environ 400 ans, probablement à la suite de modifications de l'hydrologie de la partie amont du ruisseau de Mouresse. Les données polliniques traduisent des apports lointains et révèlent un paysage asylvatique antérieurement aux reboisements RTM de la fin du $19^{\mathrm{e}}$ siècle.
\end{abstract}

Mots-clés : travertin, empreintes de feuilles, pollen, feu, Pré-Alpes françaises, Holocène, reboisement, pin noir d'Autriche (Pinus nigra subsp. nigra)

\section{ABSTRACT}

NEW DATA ON THE HOLOCENE VEGETATION IN THE SOUTHERN ALPS: THE FOSSIL FLORA OF THE SERRE DE MONTDENIER TRAVERTINE (ALPES DE HAUTE PROVENCE, FRANCE)

The travertine system of the Serre de Montdenier (alt. $1200 \mathrm{~m}$ ) presents formations containing plant macroremains (plant imprints and charcoals) and an alkaline minerotrophic peatland (fen). The analysis of travertine sequences dated by U/Th and ${ }^{14} \mathrm{C}$ allowed us to reconstruct the composition and the structure of the local ligneous flora at the beginning of the Holocene. The landscape of the Mouresse valley was then formed by a mosaic of mesophilous deciduous oak forests, of open zones with Amelanchier ovalis and Berberis vulgaris, and of riparian forests with Alnus glutinosa, Salix spp. and Vitis vinifera subsp. sylvestris. Most of these species have disappeared from the local vegetation, presently characterized by matorrals of Buxus sempervirens and afforestations of Pinus nigra subsp. nigra. The fen covering the higher part of the travertine system developed ca. 400 years ago, probably as a result of changes in the hydrology of the higher part of the Mouresse stream. Pollen data reveal a long transport and an asylvatic landscape prior to the RTM reforestation of the end of the $19^{\text {th }}$ century.

Key words: travertine, leaf imprints, pollen, wildfire, French Pre-Alps, Holocene, plantation, Pinus nigra subsp. nigra

\section{1 - INTRODUCTION}

Le bassin méditerranéen est considéré comme l'un des grands centres mondiaux de la biodiversité végétale (Davis et al., 1994 ; Quézel, 1995). Le développement des activités humaines au cours des derniers millénaires a considérablement modifié la végétation et la répartition des espèces (Beaulieu et al.,
1987 ; Barbero et al., 1990 ; Quézel \& Médail, 2003). Dans le sud de la France, la reforestation des jachères suite à la déprise agricole de la fin du $19^{\text {ème }}$ siècle, ainsi que les nombreuses plantations forestières réalisées depuis pour protéger les sols de l'érosion (Restauration des Terrains en Montagne : RTM), ont notamment contribué à perturber profondément les écosystèmes forestiers. La prise de conscience, d'abord écologique puis

\footnotetext{
${ }^{1}$ Centre de BioArchéologie et Ecologie, UMR 5059, Université de Montpellier 2, Institut de Botanique, 163 rue A. Broussonet, 34090 Montpellier, France

${ }^{2}$ MMSH, UMR 6636, 5 rue du Château de l'Horloge, 13094 Aix-en-Provence, France

${ }^{3}$ Institut des Sciences de l'Evolution, UMR 5554, Université de Montpellier 2, place E. Bataillon, 34095 Montpellier, France
} 
politique, s'est traduite par l'élaboration récente de directives internationales sur la conservation de la biodiversité (conférences de Rio en 1992 et de Paris en 2005). Il devient en effet urgent d'élaborer et de mettre en place des stratégies centrées sur la prévention et la conservation des espèces et basées sur des études scientifiques pluridisciplinaires.

Les compositions et structures passées de la végétation peuvent servir de référence, en témoignant en particulier de la paléodiversité des écosystèmes végétaux avant l'emprise de l'Homme. Les études paléoécologiques, généralement mises en oeuvre pour révéler les phénomènes climatiques ou anthropiques impliqués dans l'évolution de la végétation, permettent également d'évaluer la paléorichesse végétale qui peut être considérée comme une première approximation de la paléobiodiversité (Odgaard, 1999).

Les flores et végétations holocènes des Alpes françaises du Sud sont aujourd'hui relativement bien connues, grâce aux travaux de Beaulieu (1977), Wegmüller (1977), Coûteaux (1982), Clerc (1988), Talon (1997), Fauquette \& Talon (1995), Nakagawa (1998), Muller et al. (2000), Pothin (2000) et Ali et al. (2005). Dans la région méditerranéenne française, les archives naturelles sont plus rares : quelques études ont été réalisées depuis une trentaine d'années dans les étangs littoraux (Triat-Laval, 1979, 1982 ; Planchais \& Parra Vergara, 1984), dans des marais (Triat, 1975; Nicol-Pichard, 1987 ; Nicol-Pichard \& Dubard, 1988 ; Andrieu-Ponel et al., 2000), ainsi que dans des travertins (Ali et al., 2003). Ces travaux ont permis de mettre en évidence la dégradation générale des écosystèmes forestiers caducifoliés au profit des matorrals et des pinèdes. Les données disponibles ne permettent toutefois pas encore d'avoir une idée précise des structures et de la composition des végétations passées des régions méditerranéennes françaises.

Nous présentons ici l'étude de la macroflore et de la flore pollinique du système travertineux de Mouresse, situé sur le Serre de Montdenier (Alpes de Haute-Provence, France), dont la richesse en empreintes végétales permet de rendre compte de la flore et de la végétation ligneuses de ce massif au début de l'Holocène. Ce travail s'inscrit dans un programme d'étude des systèmes travertineux de moyenne et de haute montagne du sud-est de la France dont le principal objectif est de caractériser l'incidence des variations climatiques et des activités anthropiques sur la structuration et la dynamique de la végétation au cours de l'Holocène (Ali et al., 2003, 2005). L'étude pollinique d'une tourbière minérotrophe développée sur la partie supérieure du massif de travertin a été réalisée dans le but d'apporter des données complémentaires à l'étude macrofossile. L'enregistrement pollinique s'est avéré ne recouvrir que les quatre derniers siècles : il est susceptible de rendre compte des modifications récentes de la végétation du vallon et d'informer sur les modalités de l'initiation et du développement de la tourbière, qui abrite une espèce protégée au niveau national (Carex limosa L.).

\section{2 - ZONE D'ÉTUDE}

Situé dans les Préalpes calcaires, à proximité du canyon du Verdon, le Serre de Montdenier culmine à 1750 m d'altitude (fig. 1). Le climat régional, de type méditerranéen, est caractérisé par de forts contrastes saisonniers: les précipitations annuelles moyennes sont comprises entre 700 et $900 \mathrm{~mm}$, avec un maximum automnal ( $90 \mathrm{~mm}$ en moyenne en octobre) et une saison sèche estivale (minimum de $41 \mathrm{~mm}$ en moyenne en juillet). La moyenne annuelle des températures est de $13^{\circ} \mathrm{C}$ (données de la station météorologique de MoustiersSainte-Marie, alt. $635 \mathrm{~m}$ ).

La végétation du massif appartient à l'étage supraméditerranéen, dominé en région méditerranéenne française par la chênaie caducifoliée à Quercus pubescens et par ses faciès de dégradation (pinèdes de Pinus sylvestris et matorral à Buxus sempervirens, essentiellement). Au cours du dernier siècle, de nombreuses plantations de Pinus nigra subsp nigra ont été réalisées dans cette zone, dans le cadre du programme de Restauration des Terrains en Montagne (RTM) à des fins de sylviculture et de protection des sols contre l'érosion.

Le vallon de Mouresse (435'N, 6 $\left.{ }^{\circ} 15^{\prime} \mathrm{E}\right)$, au sein duquel s'est formé le système travertineux, incise le Serre de Montdenier vers le sud-ouest en direction du plateau de Valensole. Les versants du massif sont couverts par des matorrals à buis (Buxus sempervirens), résultant vraisemblablement, comme partout en Méditerranée septentrionale, de l'action conjuguée depuis plusieurs millénaires du pâturage et du feu (Quézel \& Médail, 2003). Ces formations végétales ouvertes abritent, outre le buis, de nombreuses espèces ligneuses pour la plupart arbustives : Amelanchier ovalis, Acer campestre, Cotoneaster integerrima, Crataegus monogyna, Genista cinerea, Juniperus phoenicea, Pinus sylvestris, Pistacia terebinthus, Prunus mahaleb, Quercus pubescens, Rosa canina, Sorbus aria et Ulmus glabra. La présence de jeunes individus de chênes blancs (Quercus pubescens) atteste toutefois la dynamique forestière actuelle de ces matorrals, qui devraient théoriquement évoluer vers des chênaies caducifoliées

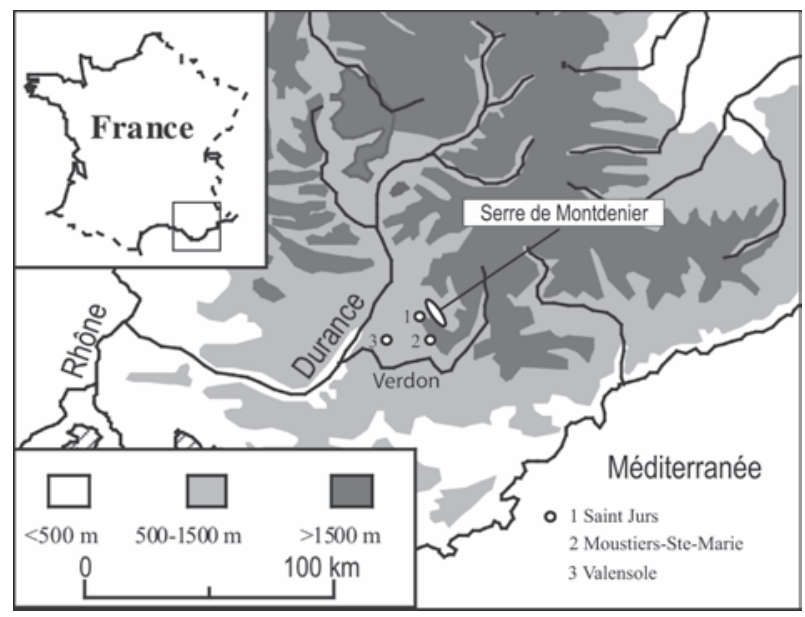

Fig. 1 : Carte de situation.

Fig. 1 : Location map. 
telles que celles que l'on peut encore observer près du village de St Jurs, au pied du massif.

Les rives du ruisseau de Mouresse sont occupées par une ripisylve discontinue constituée de saules (Salix alba, S. caprea, S. cinerea, S. eleagnos, S. purpurea), de noisetiers (Corylus avellana), de sureaux (Sambucus nigra) et de quelques peupliers blancs (Populus alba).

Quatre édifices travertineux se sont développés sur le trajet du cours d'eau (Nevière, 1996). Le plus haut (S0), situé à environ 1300 m d'altitude, est aujourd'hui recouvert par un complexe tourbeux minérotrophe alcalin à Carex paniculata, C. davalliana, C. limosa (espèce protégée au niveau national), Molinia caerulea et Schoenus nigricans. Les trois massifs encore visibles actuellement ont été nommés S1, S2 et S3. Le massif S1, édifié à $1260 \mathrm{~m}$ d'altitude au niveau d'une rupture de pente, constitue un barrage aujourd'hui incisé par le cours d'eau. Le massif S2 (1200 m), extrêmement riche en empreintes foliaires, a fait l'objet de l'étude dont les résultats sont présentés ici. Enfin, le massif S3 est développé dans la partie inférieure du vallon, à 1025 m d'altitude.

\section{3 - STRATIGRAPHIE ET ÂGE}

Les dépôts travertineux $\mathrm{S} 1$ et $\mathrm{S} 2$ sont composés de faciès construits homogènes, sans niveaux détritiques, ce qui traduit des conditions environnementales relativement stables pendant le processus de travertinisation. Par contre le dépôt inférieur S3 est constitué à la fois de niveaux construits et de faciès détritiques qui témoignent de phases d'érosion.

Le $\log$ stratigraphique de la séquence S2 (fig. 2) montre une alternance de faciès construits à empreintes et de petits niveaux crayeux contenant de rares fragments de charbons de bois. Trois fragments de travertins ont été datés par ${ }^{234}$ Uranium/230 Thorium (IFMGEOMAR, Kiel, Allemagne). D'autre part, deux charbons de bois (niveaux 6 et 10) ont donné des dates AMS ${ }^{14} \mathrm{C}$ (Poznan, Pologne) de même qu'un fragment de bois prélevé à la base de la carotte provenant de la tourbière. Nous disposons également d'une date ${ }^{14} \mathrm{C}$ qui avait déjà été obtenue dans la séquence S3 (Nevière, 1996). L'ensemble des résultats est regroupé dans le tableau 1.

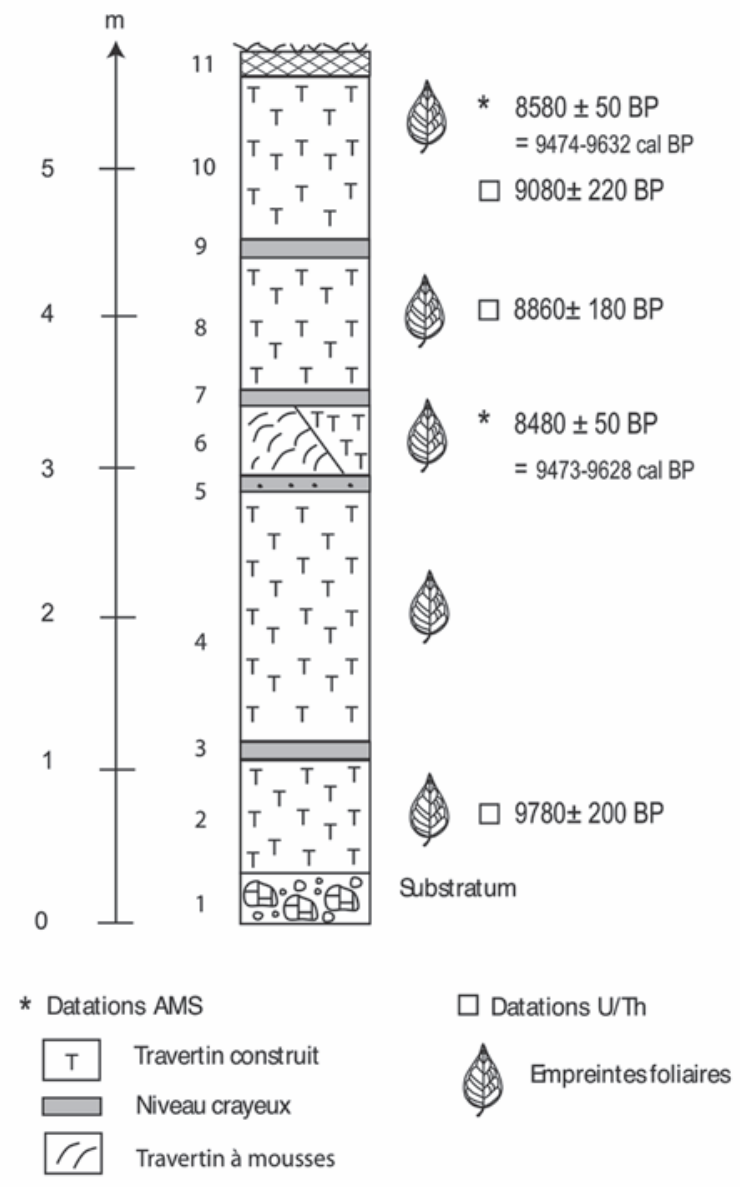

Fig. 2 : Stratigraphie de la séquence $\mathbf{S 2}$.

Fig. 2 : Stratigraphy of the S2 sequence.

\section{4 - MATÉRIELS ET MÉTHODES}

Les blocs contenant des empreintes et des charbons ont été extraits à l'aide de massettes et de burins puis les fossiles dégagés au laboratoire. Les mieux préservés ont été photographiés ou dessinés à l'aide d'un microscope stéréoscopique équipé d'une chambre claire (fig. 3). Les identifications ont été réalisées par comparaison avec des échantillons d'herbier (collection de l'UMR 5059 et de l'Herbier de l'Université de Montpellier). Les caractères de la morphologie foliaire permettent en général d'obtenir une identification au niveau de l'espèce. Par contre, en ce qui concerne

\begin{tabular}{lllllc}
\hline Echantillons & Matériel & Lab. no. & Age & Méthodes & Age cal. BP. (2 $\sigma)$ \\
\hline PBL1 & Bois & Poz-10802 & $340 \pm 30 \mathrm{BP}$ & AMS & $310-480$ \\
\hline S2L10 & Charbon & Poz-5069 & $8580 \pm 50 \mathrm{BP}$ & AMS & $9470-9630$ \\
\hline S2L6 & Charbon & Poz-5068 & $8480 \pm 50 \mathrm{BP}$ & AMS & $9470-9630$ \\
\hline S3L2 & Charbon & Gif-8930 & $7760 \pm 80 \mathrm{BP}$ & Conventionnel & $8390-8760$ \\
\hline S2L2 & Calcite & IFM-GEO & $9780 \pm 200 \mathrm{BP}$ & U/Th & $9580-9980$ \\
\hline S2L8 & Calcite & IFM-GEO & $8860 \pm 180 \mathrm{BP}$ & U/Th & $8680-9040$ \\
\hline S2L10 & Calcite & IFM-GEO & $9080 \pm 220 \mathrm{BP}$ & U/Th & $8860-9300$ \\
\hline
\end{tabular}

Tab. 1 : Datations U/Th et ${ }^{14} \mathrm{C}$ AMS

Tab. 1: U/Th and ${ }^{14} C$ AMS dates. 


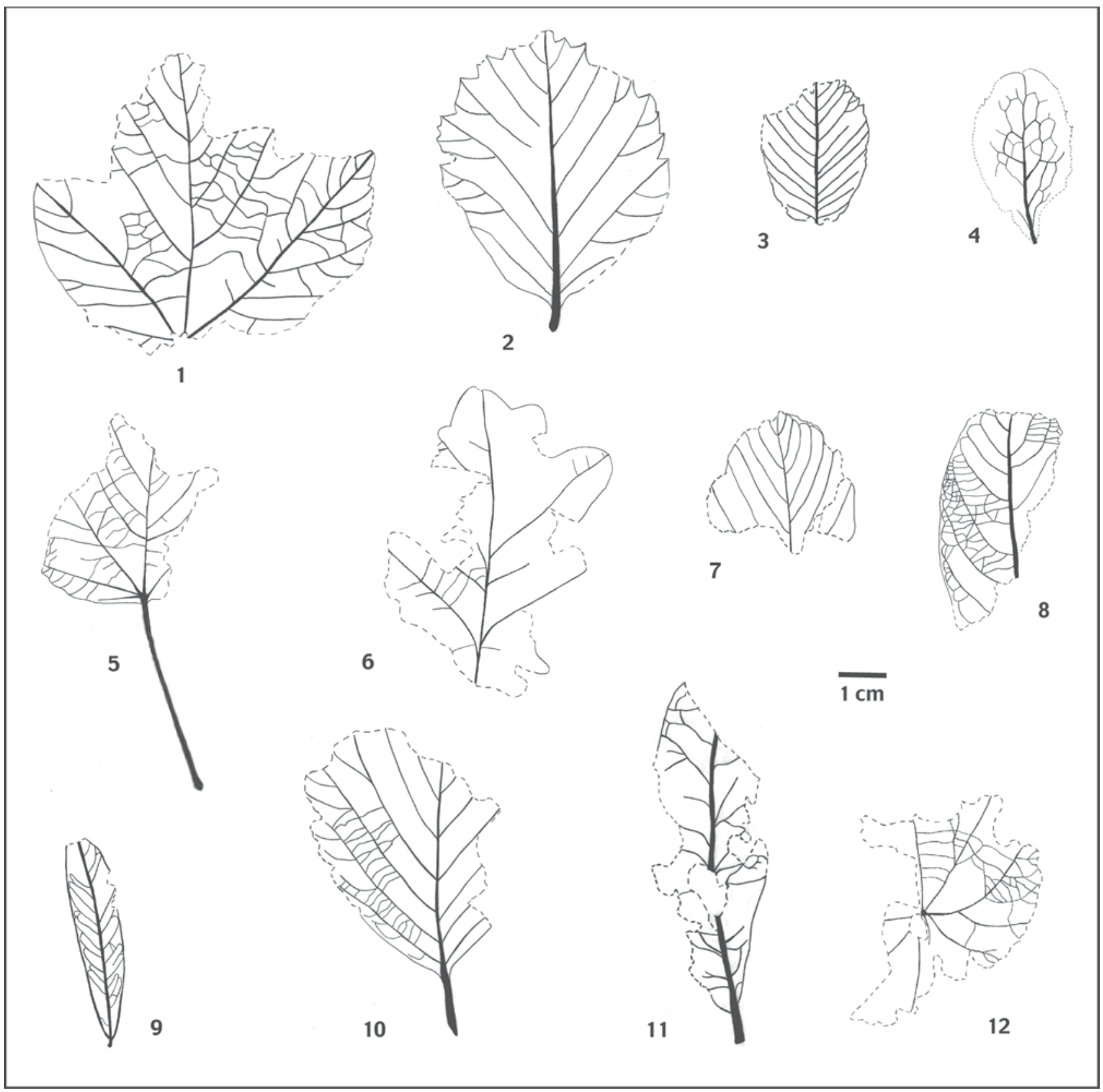

Fig. 3 : Feuilles fossiles de la séquence S2 du travertin de Mouresse. Fig. 3 : Fossil leaves of S2 Mouresse travertine
$1:$ Acer opalus
7: Rhamnus alpina
$2:$ Alnus glutinosa
8: Salix caprea
3: Amelanchier ovalis
9: Salix purpurea
4: Berberis vulgaris
10: Sorbus aria
5 : Populus alba
11 : Tilia $s p$.
6: Quercus pubescens
12 : Vitis vinifera subsp. sylvestris

l'identification de la cinquantaine de charbons de bois récupérés au sein du travertin, les caractères anatomiques n'ont le plus souvent autorisé qu'une identification au niveau du genre.

Il en est de même pour l'analyse palynologique, souvent limitée à la famille ou au genre. Les comptages polliniques ont été réalisés sur des échantillons sédimentaires de $1 \mathrm{~cm}^{3}$, extraits sur une carotte prélevée au carottier russe et préalablement traités chimiquement selon la méthode conventionnelle (Faegri \& Iversen, 1989).

\section{5 - RÉSULTATS}

En dépit d'une légère inversion chronologique corrigée par l'intervalle d'incertitude (entre L8 et L10), les dates U/Th indiquent que la séquence travertineuse S2 s'est développée au début de l'Holocène pendant une période d'environ mille ans. Bien que provenant de niveaux différents, les deux charbons de la séquence S2 donnent des âges quasiment identiques et correspondent vraisemblablement au même épisode de feu intervenu vers 9500 cal. BP. Ceci montre que la durée du 
transport des charbons depuis une même zone de feu jusqu'au ruisseau peut être très variable, certains restant enfouis dans la litière ou le sol beaucoup plus longtemps que d'autres. Ces charbons isolés inclus dans des faciès travertineux sont en conséquence susceptibles d'entraîner des incohérences chronologiques. A contrario, les charbons de bois regroupés et stratifiés dans des niveaux détritiques intercalés entre des faciès construits constituent un meilleur matériel pour réaliser des datations ${ }^{14} \mathrm{C}$, bien que là aussi des remaniements soient possibles.

Les charbons de bois extraits des niveaux 6,8 et 10 de la séquence $\mathrm{S} 2$ sont de petite taille et très friables. Leur étude anatomique a toutefois permis d'identifier :

Niveau 10 : 15 Quercus caducifolié ; 1 Monocotylédone, 5 indéterminés

Niveau 8:6 Pinus type sylvestris

Niveau 6:12 Quercus caducifolié ; 2 Pinus type sylvestris, 4 Dicotylédone, 5 charbons calcifiés indéterminables.

L'échantillonnage des empreintes foliaires a permis de dégager environ 300 spécimens, le plus souvent fragmentaires. Une vingtaine de taxons dont la liste est indiquée dans le tableau 2, ont été identifiés. Il s'agit uniquement d'espèces ligneuses car les feuilles des espèces herbacées ne sont généralement pas assez coriaces et se décomposent avant d'avoir pu laisser une empreinte.

Les taxons les mieux représentés sont Quercus pubescens, Tilia sp., Amelanchier ovalis, Acer opalus, Alnus glutinosa, Salix purpurea et Salix eleagnos. Des aiguilles et des charbons de bois de Pinus type sylvestris ont aussi été identifiés dans les niveaux supérieurs. La comparaison avec la flore actuelle du vallon de Mouresse montre que plusieurs espèces présentes au début de l'Holocène (Acer opalus, Alnus glutinosa, Berberis vulgaris, Rhamnus alpina, Tilia sp. et Vitis vinifera subsp. sylvestris) ne sont pas retrouvées dans la flore locale. Inversement d'autres espèces ligneuses qui se développent aujourd'hui à proximité du travertin ne sont pas représentées dans la flore fossile.

La tourbière a une origine beaucoup plus récente (environ 400 ans) et recouvre une ancienne zone travertineuse (S0) qui a peut-être fonctionné jusqu'à cette époque. Les dépôts stériles compris entre 175 et $110 \mathrm{~cm}$ sont vraisemblablement issus d'une ou de plusieurs phases érosives du travertin en amont. La présence de fragments de travertin au sein de l'ensemble de la séquence indique la permanence des apports détritiques. Le spectre pollinique de surface met enfin en

\begin{tabular}{|c|c|c|c|c|c|}
\hline Taxons & Niveau 2 & Niveau 4 & Niveau 6 & Niveau 8 & Niveau 10 \\
\hline \multicolumn{6}{|l|}{ Gymnospermes } \\
\hline Pinus sylvestris-type & & & $\mathrm{c}$ & $\mathrm{c},++$ & ++ \\
\hline \multicolumn{6}{|l|}{ Dicotylédones } \\
\hline Acer campestre & & & & + & \\
\hline Acer opalus & ++ & ++ & +++ & ++ & \\
\hline Alnus glutinosa & & ++ & & & \\
\hline Amelanchier ovalis & ++ & ++ & & & \\
\hline Berberis vulgaris & & + & & & \\
\hline Populus alba & & & & & + \\
\hline Quercus caducifolié & & & $\mathrm{c}$ & & $\mathrm{c}$ \\
\hline Quercus pubescens & & ++ & ++ & & ++ \\
\hline Quercus robur & & & + & & + \\
\hline Rhamnus alpina & & & & & + \\
\hline Rhamnus frangula & & + & & & \\
\hline Rubus sp. & & + & & & \\
\hline Salix caprea & & & & + & \\
\hline Salix cinerea & & & & + & \\
\hline Salix eleagnos & ++ & + & & & \\
\hline Salix fragilis & & & + & & \\
\hline Salix purpurea & & ++ & & & \\
\hline Sorbus aria & + & + & ++ & & \\
\hline Tilia sp. & ++ & & +++ & +++ & ++ \\
\hline Vitis vinifera subsp. sylvestris & & & & + & \\
\hline \multicolumn{6}{|l|}{ Monocotylédones } \\
\hline cf. Phragmites & & & + & + & + \\
\hline Nombre d'empreintes & 77 & 122 & 39 & 36 & 23 \\
\hline
\end{tabular}

Tab. 2 : Liste des espèces identifiées dans la séquence travertineuse $\mathbf{S 2}$ du vallon de Mouresse.

+ présence ; ++ fréquent ; +++ abondant ; c charbon

Tab. 2: List of taxa identified within S2 travertine sequence at Mouresse.

+ presence $;++$ frequent $;+++$ abundant $;$ c charcoal 
évidence la prédominance du pollen de Pinus type sylvestris, qui marque les reboisements récents en Pinus nigra subsp. nigra.

\section{6 - DISCUSSION}

\section{1 - DYNAMIQUES PALÉOENVIRONNEMENTALES DU DÉBUT DE L'HOLOCÈNE}

La séquence S2 du travertin de Mouresse témoigne de l'existence entre 10000 et 9000 cal. BP d'une flore ligneuse différente de l'actuelle. La présence passée de taxons héliophiles comme Amelanchier, Rubus et Berberis et d'espèces forestières comme Quercus pubescens, Acer opalus et Tilia sp. indique une végétation en mosaïque en relation avec le sol ou l'exposition. Les pentes rocheuses devaient être colonisées par Pinus type sylvestris, Berberis vulgaris, Rubus sp. et Amelanchier ovalis, les abords du ruisseau par Alnus glutinosa, Salix purpurea, Salix eleagnos et Populus alba, tandis que Quercus pubescens, Acer opalus, Sorbus aria et Tilia constituaient probablement des peuplements forestiers sur sols profonds.

Le développement et la suprématie de la chênaie caducifoliée mixte à partir du Boréal est un phénomène bien connu dans le sud-est de la France, depuis les Alpes méridionales (Beaulieu, 1977 ; Digerfeldt et al., 1997 ; Nakagawa, 1998) jusqu'au plaines provençales (Triat-Laval, 1979 ; Nicol-Pichard, 1987 ; Nicol-Pichard \& Dubard, 1988). Les données obtenues au Serre de Montdenier mettent en évidence la complexité des paysages des Alpes provençales au début de l'Holocène, qui apparaissent caractérisés par une mosaïque de zones forestières et de zones ouvertes. L'abondance des charbons de bois permet de supposer un rôle important des incendies dans le maintien de cette hétérogénéité paysagère. Les empreintes foliaires de la séquence S2 attestent en outre la présence locale de Tilia entre 10000 et 9000 cal. BP, et confirme les études palynologiques régionales qui datent l'apparition du tilleul vers 10500 ans cal. BP et son développement optimal aux alentours de 9000 cal. BP (Beaulieu, 1977 ; Digerfeldt et al., 1997 ; S.D. Muller, données non publiées).

Des informations plus récentes sur l'évolution du paysage sont fournies par la séquence aval (S3), malheureusement dépourvue d'empreintes végétales (fig. 4). La base de ce dépôt a livré des charbons de bois datés d'environ 8600 cal. BP, associés à un dépôt sablodétritique (Nevière, 1996). Ces charbons témoignent d'un ou de plusieurs épisodes de feu ayant potentiellement ouvert le milieu et favorisé l'érosion des versants. Les blocs de calcaire et de travertins présents dans la partie médiane de la séquence $\mathrm{S} 3$ traduisent des phénomènes érosifs postérieurs à $8600 \mathrm{cal}$. BP et pourraient provenir de l'érosion des travertins amonts (S0, S1 et S2) qui sont fortement incisés. Il est à souligner que les deux épisodes de feu enregistrés dans les séquences S2 et S3 coïncident avec des périodes de faibles

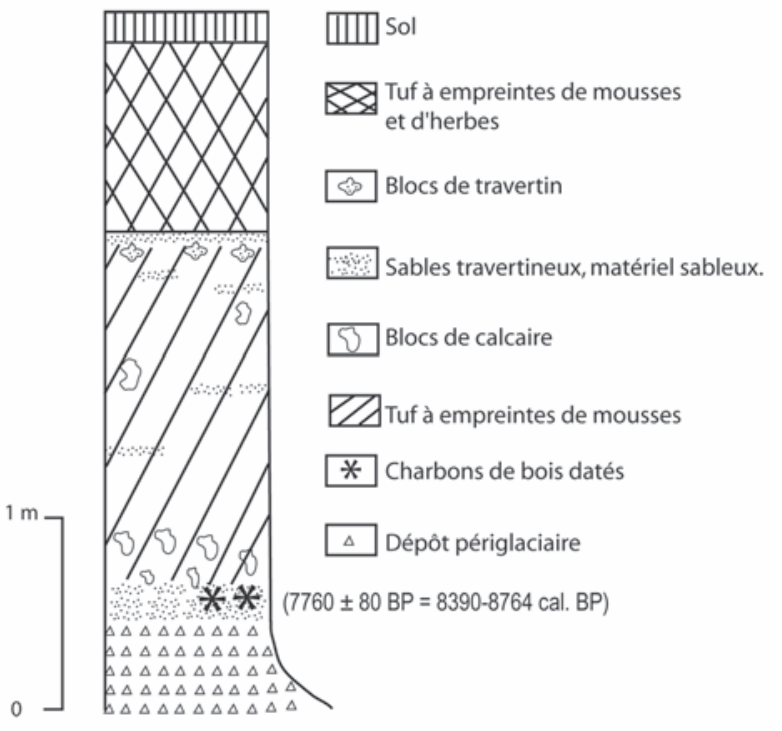

Fig. 4 : Stratigraphie de la séquence S3.

Fig. 4: Stratigraphy of the S3 sequence.

précipitations matérialisées par la baisse du niveau du lac de Saint-Léger (Digerfeldt et al., 1997).

L'absence de restes végétaux identifiables dans les différentes séquences postérieurement à 9000 cal. BP empêche toute reconstitution paléoécologique locale, notamment à propos de la mise en place du matorral actuel à Buxus sempervirens. L'analyse anthracologique réalisée à Unang dans le Vaucluse (alt. 150 m) montre que le Buis y apparaît vers 7000 cal BP, en association avec le Chêne vert et parallèlement au déclin de la chênaie caducifoliée (Thiébault, 1988). Cette dynamique paléoenvironnementale, par ailleurs observée dans l'ensemble de la région méditerranéenne (Triat, 1975 ; Heinz \& Thiébault, 1998), est interprétée comme le résultat de l'exploitation du milieu par l'Homme. Buxus n'est jamais très bien représenté dans les analyses palynologiques. Son apparition régionale est toutefois enregistrée postérieurement à $8000 \mathrm{cal}$. BP à Tourves (Nicol-Pichard, 1987) et vers 5200 cal. $\mathrm{BP}$ à Saint-Léger (Digerfeldt et al., 1997) en relation avec la régression de la chênaie mixte, ce qui confirme l'origine anthropique de son développement.

\section{2 - DYNAMIQUES PALÉOENVIRONNEMENTALES DES QUATRE DERNIERS SIÈCLES}

La tourbière minérotrophe alcaline du vallon de Mouresse est développée sur le massif travertineux le plus élevé, en dessous d'un replat actuellement colonisé par un bois de Fraxinus excelsior, dont le développement semble dater de 100-150 ans. L'origine de la zone humide est probablement liée à une modification de l'utilisation des sols en amont, qui aurait entraîné un changement de l'hydrologie du ruisseau. En dépit de son origine récente et probablement anthropique, la tourbière du vallon de Mouresse est intéressante sur le plan floristique : elle abrite en effet plusieurs espèces tourbicoles, dont le rare Carex limosa. L'enregistrement constant de plusieurs taxons indicateurs 


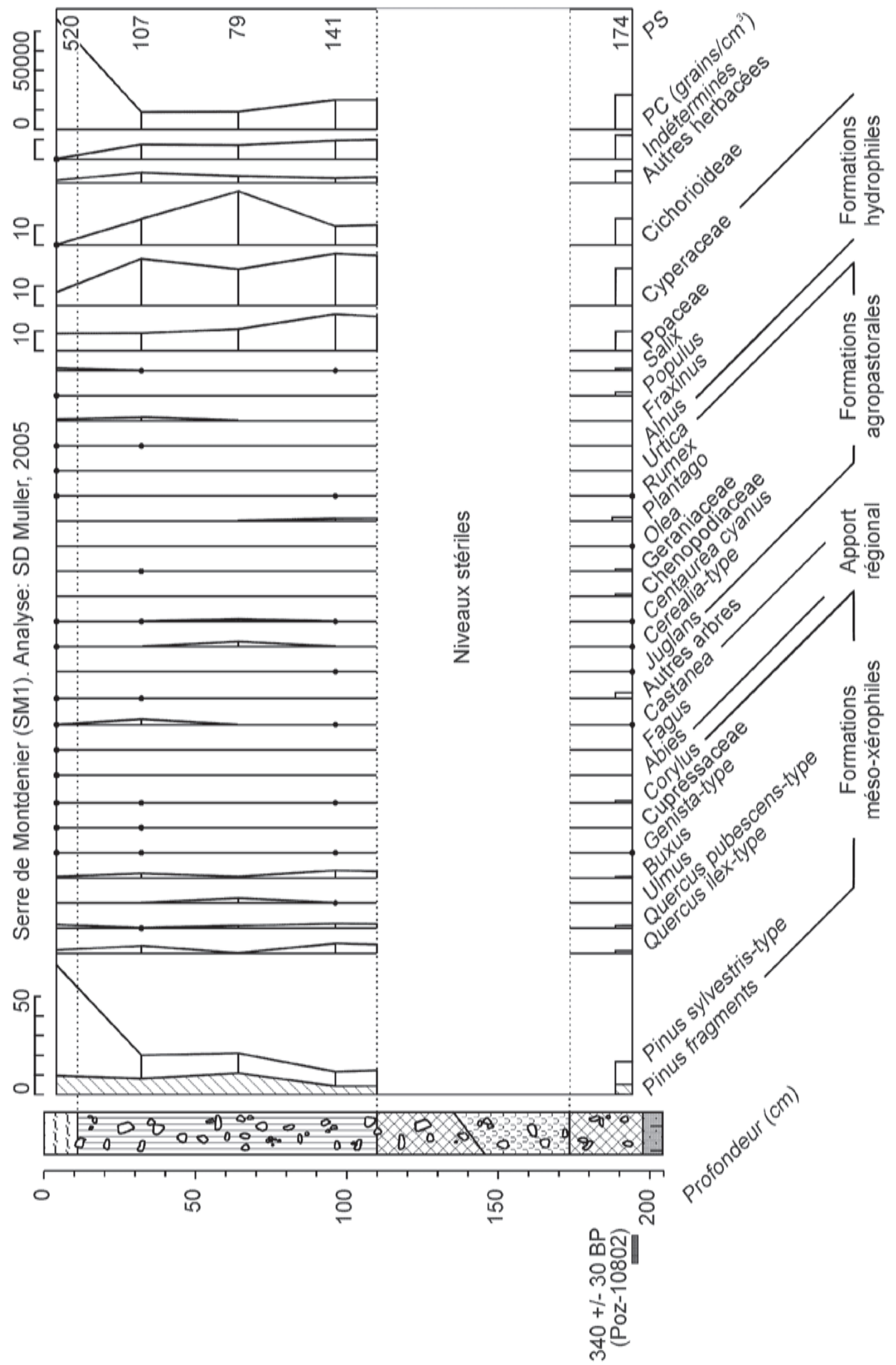


d'anthropisation (Castanea, Olea, Juglans, Plantago, Cerealia, Centaurea, Chenopodiaceae) atteste l'importance des activités agropastorales dans la région depuis au moins quatre siècles (fig. 5). Il est cependant très vraisemblable que si le pastoralisme a dû se développer sur les pentes du Serre de Montdenier, les activités agricoles devaient y être très réduites. Les pollens d'arbres cultivés présents dans la tourbière ont été transportés par le vent sur de grandes distances et pouvaient provenir du plateau de Valensole ou de zones plus éloignées. Cela est attesté en particulier par l'enregistrement pollinique du châtaignier (Castanea), espèce acidiphile et calcifuge (Rameau et al., 1993), et donc n'ayant jamais pu se développer dans la zone calcaire du Serre de Montdenier. Les peuplements importants les plus proches sont localisés sur une bande de sables et de grés du Crétacé supérieur qui traverse les bassins d'Apt et de Forcalquier, et dans la région de Banon-Vachères à environ $50 \mathrm{~km}$ à vol d'oiseau du Serre de Montdenier (Lieutaghi, 2005). Les pourcentages polliniques relativement importants de Castanea traduisent peut-être aussi les vaines tentatives pour l'introduire sur la commune de Vinon-sur-Verdon (Piégay, 1996), à environ $40 \mathrm{~km}$ au sud-ouest du Serre de Montdenier.

Le diagramme pollinique de la tourbière de Mouresse ne renseigne que peu sur les dynamiques de végétation régionales. Il témoigne cependant du caractère fortement asylvatique de la végétation avant les reboisements en pins noirs d'Autriche de la fin du $19^{\mathrm{e}}$ siècle, marqués par la hausse des pourcentages de Pinus type sylvestris dans le niveau de surface. Ces reboisements, réalisés dans le cadre des travaux de Restauration des Terrains en Montagne (RTM), ont permis la constitution de vastes peuplements de résineux, qui ralentissent fortement la régénération des chênaies caducifoliées. Par ailleurs dans les écosystèmes méditerranéens où le feu est un des facteurs écologiques majeurs (Carcaillet et al., 1997), ces reboisements en résineux très inflammables augmentent le risque de destruction de la végétation par des incendies de grande ampleur.

\section{7 - CONCLUSION}

L'étude du système travertineux du vallon de Mouresse a permis de caractériser la flore diversifiée qui se développait sur le Serre de Montdenier au début de l'Holocène. Les empreintes foliaires permettent de reconstituer un paysage hétérogène, caractérisé par des zones de chênaies mixtes caducifoliées, des milieux ouverts au moins en partie liés à l'occurrence d'incendies et des ripisylves bordant les ruisseaux. A l'heure actuelle, une végétation ouverte dominée par le buis a remplacé les écosystèmes forestiers. Plusieurs espèces caducifoliées initialement présentes (Acer opalus, Alnus glutinosa, Berberis vulgaris, Rhamnus alpina, Tilia sp. et Vitis vitifera subsp. sylvestris) ont aujourd'hui disparu de la flore locale, vraisemblablement à l'issue de l'ouverture anthropique du milieu.
L'abandon des activités agropastorales depuis la fin du $19^{\mathrm{e}}$ siècle pourrait favoriser le retour de boisements à chênes pubescents et tilleuls. L'introduction massive du Pin noir d'Autriche, espèce envahissante, risque d'entraver cette dynamique naturelle, perceptible dans de nombreuses zones méditerranéennes sur sol profond.

Les résultats présentés ne révèlent l'histoire de la végétation locale que sur de courtes périodes de temps, au début et à la fin de l'Holocène. Ils apportent cependant des informations importantes sur la structure et la composition de la végétation dans une région climatique peu favorable à la préservation d'archives sédimentaires. Le sud-est de la France est riche en séquences travertineuses holocènes à fort potentiel paléoécologique (Vaudour, 1994), dont l'étude est susceptible d'apporter des données complémentaires.

\section{REMERCIEMENTS}

Les auteurs remercient les deux relecteurs anonymes dont les remarques ont contribué à améliorer le texte de cet article.

\section{RÉFÉRENCES BIBLIOGRAPHIQUES}

ALI A.A., TERRAL J.F., GUENDON J.L., \& ROIRON P., 2003 Holocene palaeoenvironmental changes in Southern France : a palaeobotanical study of travertine at St-Antonin, Bouches-duRhône. The Holocene, 13, 293-298.

ALI A.A., ROIRON P., GUENDON J-L., POIRIER P., \& TERRAL J.-F., 2005 - Fire and vegetation pattern changes in the southern inner French Alps (Queyras Massif) during the Holocene : geomorphologic and charcoal analyses of travertine sequences. The Holocene, 15, 149-155.

ANDRIEU-PONEL V., PONEL P., JULL A.J.T., BEAULIEU J.-L. DE, BRUNETON H., \& LEVEAU P., 2000 - Towards the reconstruction of the Holocene vegetation history of Lower Provence : two new pollen profiles from Marais des Baux. The Holocene, 10, 341-355.

BARBERO M., BONIN G., LOISEL R., \& QUÉZEL P., 1990 Changes and disturbances of forest ecosystems caused by human activities in the western part of the Mediterranean basin. Vegetatio, 87, 151-173.

BEAULIEU J.-L. DE, 1977 - Contribution pollenanalytique à l'histoire Tardiglaciaire et Holocène de la végétation des Alpes méridionales françaises. Thèse Université Aix-Marseille 3, 358 p.

BEAULIEU J.-L. DE, RICHARD H., RUFFALDI P., \& CLERC J., 1994 - History of vegetation, climate and human action in the french Alps and the Jura over the last 15,000 years. Dissertationes Botanicae, 234, 253-275.

CARCAILlET C., BARAKAT H. N., PANAÏOTIS C., \& LOISEL R., 1997 - Fire and late Holocene expansion of Quercus ilex and Pinus pinaster in Corsica. Journal of Vegetation Science, 8, 85-94.

CLERC J., 1988 - Recherches pollenanalytiques sur la paléoécologie tardiglaciaire et holocène du Bas-Dauphiné. Thèse Université Aix-Marseille 3, 180 p.

COUTEAUX M., 1982 - Recherches pollenanalytiques en Oisans : le plateau de Brande (Alpes d'Huez, Isère, France). Bulletin de la Société Royale de Botanique de Belgique, 115, 91-106.

DAVIS S.D., HEYWOOD V.H., \& HAMILTON A.C. (Eds.), 1994 - Centres of Plant Diversity. A Guide and Strategy for their Conservation. Vol. 1. Europe, Africa, South West Asia and The Middle East. WWF and IUCN, IUCN Publications Unit, Cambridge. 
DIGERFELDT G， BEAULIEU J.-L. DE, GUIOT J., \& MOUTHON J., 1997 - Reconstruction and paleoclimatic interpretation of Holocene lake-level changes in Lac de Saint-Leger, Haute-Provence, southeast France. Palaeogeography, Palaeoclimatology, Palaeoecology, 136 (1-4), 231-258.

FAEGRI K., \& IVERSEN J., 1989 - Textbook of Pollen Analysis. K. Faegri, P.E. Kaland \& K. Kzrywinski (éditeurs), J. Wiley \& Sons, Chichester, $328 \mathrm{p}$.

FAUQUETTE S., \& TALON B., 1995 - Histoire de la végétation forestière d'un site du Briançonnais: le Lac de Cristol (HautesAlpes, France). Comptes Rendus de l'Académie des Sciences de Paris, Série II, Sciences de la Terre, 321, 255-262

HEINZ C., \& THIEBAULT S., 1998 - Characterization and Palaeoecological Significance of Archaeological Charcoal Assemblages during Late Glacial and Post-Glacial Phases in Southern France. Quaternary Research, 50, 56-68.

LIEUTAGHI P., 2005 - Jardins du chêne blanc. Chênaie pubescente méridionale, paysages floraux, économie traditionnelle, évolution. Actes Sud, Arles, $121 \mathrm{p}$.

MULLER S.D., DAVID F., \& WICHA S., 2000 - Impact de l'exposition et de l'anthropisation sur la dynamique forestière dans les Alpes du Sud (France). Géographie physique et Quaternaire, 54, 227-239.

NAKAGAWA T., 1998 - Etudes palynologiques dans les Alpes françaises centrales et méridionales: Histoire de la végétation tardiglaciaire et holocène. Thèse Université Aix-Marseille 3, 206 p.

NEVIERE C., 1996 - Les systèmes travertineux du Serre de Montdenier (Alpes de Haute-Provence). Études de Géographie Physique, Aix-en-Provence, 25, 31-34.

NICOL-PICHARD S., 1987 - Analyse pollinique d'une séquence tardi- et postglaciaire à Tourves (Var, France). Ecologia mediterranea, 13 (1-2), 29-42.

NICOL-PICHARD S., \& DUBAR M., 1988 - Reconstruction of late-glacial and holocene environments in southeast France based on the study of a 66-m long core from Biot, Alpes Maritimes. Vegetation History and Archaeobotany, 7 (1), 11-15.

ODGAARD B.V., 1999 - Fossil pollen as a record of past biodiversity. Journal of Biogeography, 26 (1), 7-17.

PIEGAY J., 1996 - Les terroirs de Vinon et alentours, rues, sites et lieux-dits. Association d'Histoire et d'Archéologie de Vinon sur Verdon (éd.), Vinon, 173 p.
PLANCHAIS N., \& PARRA VERGARA I., 1984 - Analyses polliniques de sédiments lagunaires et côtiers en Languedoc, en Roussillon et dans la province de Castellon (Espagne) ; Bioclimatologie. Bulletin de la Société botanique de France, 131, 97-105.

POTHIN A., 2000 - Impact de l'homme dans les milieux montagnards : Analyse pollinique de la séquence du Laux (alt. : $1108 \mathrm{~m}$, Champsaur, Hautes-Alpes). Mémoire DEA Université Aix-Marseille $3,38 \mathrm{p}$.

QUÉZEL P., 1995 - La flore du bassin méditerranéen : origine, mise en place, endémisme. Ecologia mediterranea, 21, 19-39.

QUÉZEL P., \& MÉDAIL F., 2003 - Ecologie et biogéographie des forêts du bassin méditerranéen. Elsevier, $571 \mathrm{p}$.

RAMEAU J.-C., MANSION D., DUME G., LECOINTE A., TIMBAL J., DUPONT P., \& KELLER R., 1989-1993 - Flore forestière française, Guide écologique illustré, 2 tomes. Institut pour le Développement forestier, Ministère de l'Agriculture et de la Pêche, Direction de 1'Espace rural et de la Forêt, Ecole nationale du Génie rural, des Eaux et des Forêts, Paris, 4206 p.

THIÉBAULT S., 1988 - L'Homme et le milieu végétal : analyses anthracologiques de six gisements des Préalpes au Tardi- et au Postglaciaire. Documents d'Archéologie Française, 15, 110 p.

TALON B., 1997 - Evolution des zones supra-forestières des Alpes sud-occidentales françaises au cours de l'Holocène. Analyse pédoanthracologique. Thèse Université Aix-Marseille 3, 175 p.

TRIAT H., 1975 - L'analyse pollinique de la tourbière de Fos-surMer (B. du Rh.). Ecologia mediterranea, 1, 109-121.

TRIAT-LAVAL H., 1979 - Contribution pollenanalytique à l'histoire tardi- et postglaciaire de la végétation de la basse vallée du Rhône. Thèse Université Aix-Marseille 3, 343 p.

TRIAT-LAVAL H., 1982 - Pollenanalyse de sédiments quaternaires récents du pourtour de 1'Etang de Berre. Ecologia mediterranea, 8, 97-115.

VAUDOUR J., 1994 - Evolution holocène des travertins de vallée dans le Midi Méditerranéen français. Géographie physique et Quaternaire, 48, 315-326.

WEGMULLER S., 1977 - Pollenanalytische Untersuchungen zur spät- und postglazialen Vegetationsgeschichte der französischen Alpen (Dauphiné). P. Haupt, Bern, 185 p. 